\title{
Polyurethane foam behaviour during impact
}

\author{
Stanislav Špirk ${ }^{1, *}$, Michal Křǐžek ${ }^{1}$, Štěpán Jeníček ${ }^{1}$ \\ ${ }^{1}$ University of West Bohemia in Pilsen, Regional Technological Institute, Univerzitní 22, 30614 \\ Plzeň, Czech Republic
}

\begin{abstract}
This contribution is focused on the passive safety of the railway vehicles interiors. The passive safety has become a new area of the rail vehicles design. In the field of rail vehicles interior passive safety the computer simulations are most widely used. The computer simulations must be based on relevant material data. For some common materials (eg. steel, aluminium, etc.) the material data are ascertainable. The interiors of rail vehicles contain quite specific materials frequently. One of the material with more complicated mechanical properties is polyurethane foam (PUR). The aim of this paper is to present validation of material parameters for specific polyurethane foam by experiment. The material properties (for seat made from Elastoflex W 5662 covered with Holdsworth BHLH 31634) will be useful for further simulations.
\end{abstract}

Keywords: Crash, foam, PUR, dynamics, Elastoflex, Holdsworth

\section{Introduction}

Mechanical properties of polymer foams at ambient temperatures have been evaluated by various authors using experiments with static and shock loading. Their results are available in studies [1] and [2]. Here, foam parts are considered an important element of a vehicle interior that can protect passengers from severe deceleration by gradual and controlled absorption of energy. An optimal material should be able to absorb kinetic energy of impact and have strength below a relevant force limit.

Passenger injury is a result of the collision between a body part and the interior feature impacted. In case of collision with a padded seat, the description of the seat crash behaviour is complicated. The main aspects are connected with the soft parts of this structure. The final mechanical behaviour during impact will be the result of the response of PUR prestressed by the cover fabric. The prestress of PUR is significant but difficult to measure. The best way of material properties validation is to conduct a test which is close to the conditions during the actual passenger impact. The foam material Elastoflex W 5662 covered with Holdsworth BHLH 31634 cloth was used in experiment. The material model presented in the end of paper has the good concordance with experiment.

\footnotetext{
*Corresponding author: spirks@rti.zcu.cz

Reviewers: Mariana Pajtášová, Ksawery Szykiedans
} 


\section{Properties of foam materials}

Mechanical properties of polymer foams at ambient temperatures have been evaluated by various authors using experiments with static and shock loading. Their results are available in studies [1] and [2]. Here, foam parts are considered an important element of a vehicle interior that can protect passengers from severe deceleration by gradual and controlled absorption of energy. All investigations in this field focus predominantly on the capability of controlled energy absorption. Optimal energy-absorbing materials must absorb the kinetic energy of an impact, while keeping the reaction force below a certain limit. Energy is dissipated by cell bending, buckling or fracture. Generally, the stress-strain curve shows a long horizontal section followed by a steep rise (Fig. 1). This explains the high efficiency of energy absorption achieved with foamed materials. In addition, a foamed specimen always provides lower acceleration at maximum compressive force than a solid specimen of the foam matrix material with the same volume absorbing the same amount of energy.

Foamed materials exhibit linear response within a relatively narrow interval up to approximately $5 \%$ engineering strain. Here, the slope of the curve is given by the modulus of elasticity of the material. As the load on the foam increases, the cells begin to collapse by elastic buckling, plastic yielding or brittle crushing - depending on the mechanical properties of the specimen. The collapse continues under approximately constant stress, until the cell walls come into contact under the pressure. At this point, stress begins to rise steeply, as the material densifies. During unloading, the stress decreases non-linearly. Absorbed energy is generally represented by the area below the curve in the plot.

Upon impact, the passenger body is subjected to deceleration whose magnitude depends on the stiffness of the seat material. Consequently, the fundamental requirement is to define the dependence of true stress in a foamed material on its actual engineering strain under compression. It is usually an increasing function, whereas the unloading behaviour is defined separately through a parameter which reflects energy dissipation. Simulations were carried out using the type-45 material model (General nonlinear strainrate dependent foam with optional energy absorption) [3] (Pam-Crash software). The characteristics of PUR foam can be found in literature [4]. Figure 1 shows the static true stress-true strain plot for PUR.

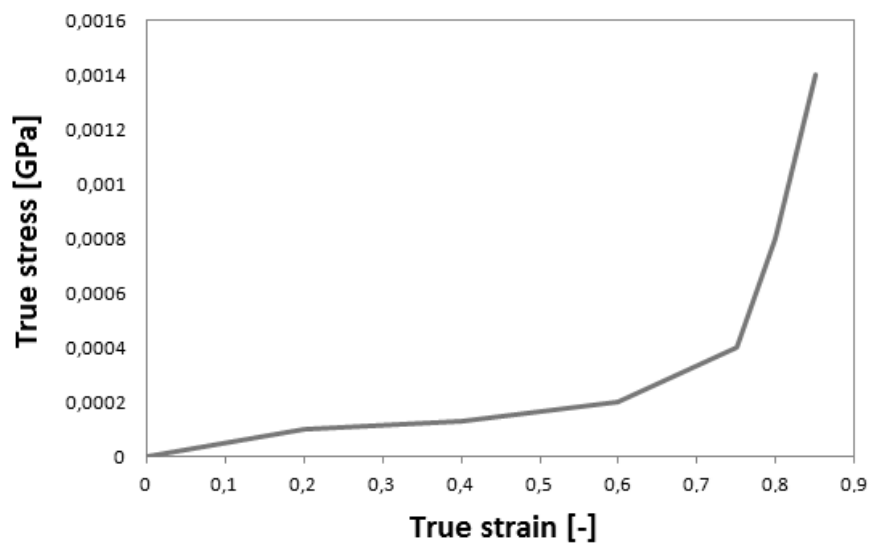

Fig. 1. Initial stress-strain dependence of the foam material (PUR)

The key question is whether the literature data offer an accurate characterization of the behaviour of the seat material. Additional factors may play a role in a real-world seat, such as foam pre-stress. Although the thickness of the cover fabric can be considered negligible, the fabric will still apply certain pre-stress to the filling. Consequently, the mechanical 
properties of the seat cushion never quite match the properties of the filling material alone. Clearly, a tension test cannot be carried out here. Foamed materials are typically tested in compression. However, testing a polyurethane foam seat (including the cover fabric) at higher loading rates in a conventional testing machine is complicated and difficult to carry out. Since some preliminary materials data is already known, the best course of action appears to involve a simple experiment to verify the definition of the material.

\section{Experimental validation of mechanical properties of foamed material}

The purpose of the simple experiment is to verify the behaviour of an actual material under conditions (loading mode, load rate, etc.) which are as close as possible to the real-world situation. In general, calculations are best validated by experiments. Various collision scenarios involve the contact between various parts of human body and the seat. Head impact is unquestionably considered the most important one. An experiment which is intended to verify the behaviour of a material should therefore follow this scenario. From the standpoint of calculation validation, a head-seat impact makes the ideal experiment. If its results are also to be used for validating a foamed material, there is a problem with a vast number of unknown and difficult-to-verify parameters. The outcome would be affected by the stiffness of the seat, by the properties of the material of the passenger head model, and by its geometry, weight and stiffness. In order to obtain relevant output for validating the data of the foamed material, the experiment should be simplified to involve an impact of a rigid ball on a foamed material specimen placed on a rigid plate. This approach is close to Multibody dummy head (seen eg. in [7]) which impact to the FE model part. In the simplest arrangement, the ball could be dropped on the seat cushion. However, this poses an unacceptable risk of the ball falling away from the seat cushion (or bouncing away), causing an injury or damaging the measuring equipment. The most basic method of guiding the movement is to use a rotating arm. The resulting pendulum would cause the ball striker to hit the same spot repeatedly. Energy losses associated with the impact would therefore be easy to evaluate. In addition, the arm would make the transmission of data to a recording equipment very straightforward. The proposed experimental arrangement is shown in Fig. 2.
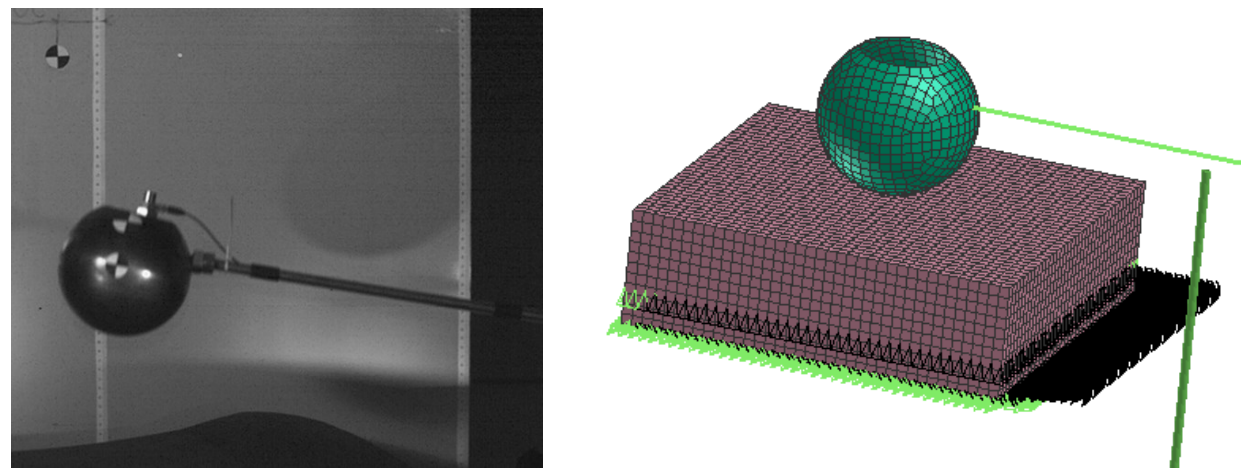

Fig. 2. Experimental set-up and simulation

The striker is a steel ball of $3 \mathrm{~mm}$ steel sheet filled with concrete for greater weight. The ball is provided with a threaded hole into which the threaded end of the rotating arm is screwed. The other end of the arm is attached to a hinge mounted to a fixed plate. The plate height is such that the ball comes into contact with the specimen at the point when the arm 
is in horizontal position. From the upper to the horizontal position, the fall can be evaluated analytically. Below that position, FEM computation should be used.

The acceleration of the ball was measured using PCB 352C33 S/N 120471 accelerometer. This is a dynamic accelerometer suitable for measuring rapidly changing accelerations. It was attached by means of a magnet. It was connected to a computer via a Highspeed USB carrier NI9234 logger. The readings were recorded by means of the Signal expres 2011 software. In order to obtain a slow-motion video sequence, a high-speed Olympus ispeed 2 camera has been used. [5] Its speed was $500 \mathrm{fps}$ at an $800 \times 600$ resolution (Fig 3). The size of the camera sensor means that more expensive photographic lenses are not required. C-mount lenses are easily attached to the camera and do not compromise image quality. For experiment was used the c-mount lense with low color aberration and low TV distortion, focal length $35 \mathrm{~mm}$, aperture 2, mod $0.35 \mathrm{~m}$, back focal length 10.14 , angle of view $14^{\circ}$ image circle $11 \mathrm{~mm}$. The station was lit with two $1500 \mathrm{~W}$ halogen lamps. The recording from the high-speed camera was processed using the Ispeed 2 Advanced PC program. The crucial task for validating material data is to verify the speed at the moment of impact of the striker. By this means, it can be confirmed whether this is a free fall case where friction of the hinge, aerodynamic drag and other aspects can be neglected. The highspeed camera can record the position of a chosen point over a period of time (where one pixel is $0.53 \mathrm{~mm}$, with software $1 / 4$ pixel approximation, accuracy average $\pm 0.63 \mathrm{~mm}$ with used optics). Hence, one can evaluate the position and the speed of the striker throughout the process.

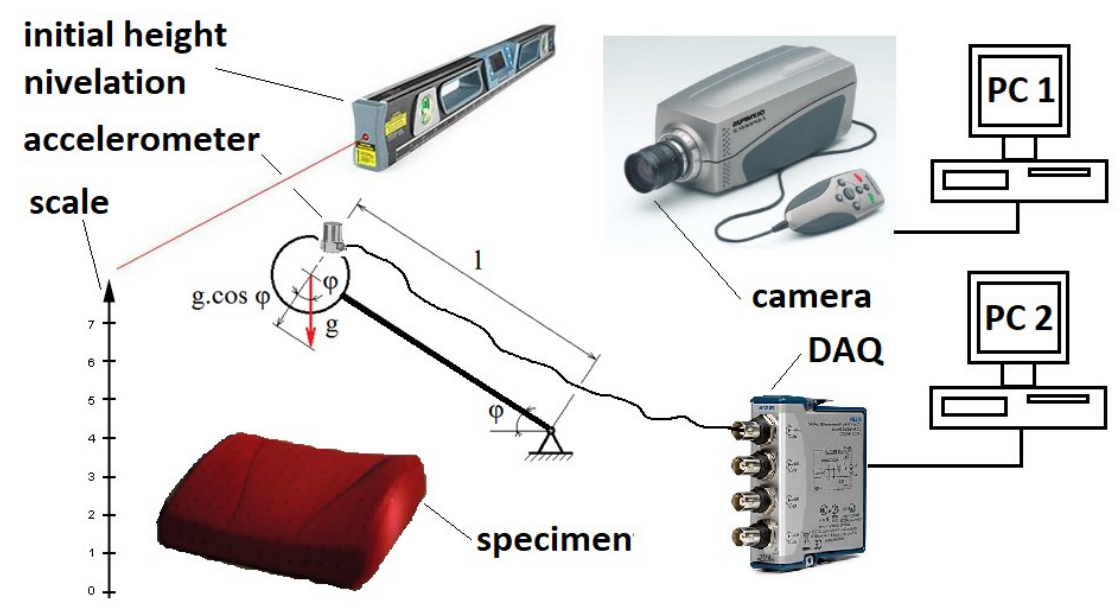

Fig. 3. Functional diagram of the test bed

If no other than the gravitational acceleration acts on the striker, its impact speed can be determined analytically. In fact, this is a special case of a pendulum. The arm, which has a length of $1=910 \mathrm{~mm}$ and a weight of $0.6 \mathrm{~kg}$, holds a striker of $\mathrm{m}=2.4 \mathrm{~kg}$. This ball-shaped striker has a radius of $60 \mathrm{~mm}$. The angle between the arm and the horizontal is denoted as $\varphi$. Several simplifying assumptions were used for solving the mathematical pendulum problem. Small displacements are not considered. Therefore, equation 1 is used for finding the displacement $\varphi$.

$$
\int_{0}^{\varphi} \frac{g}{l} \cos \varphi 2 d \varphi=\int_{0}^{\omega^{2}} d \omega^{2}
$$


For the speed $\mathrm{v}$ at the time instant when $v=0$ (striker impact), one can use the assumption that the vertical speed corresponds to the tangential speed because $\cos \varphi=1$. The resulting speed $\mathrm{v}$ at the moment of impact of the striker is given by equation 2 .

$$
v=\sqrt{2 \cdot g \cdot l \cdot \sin \varphi}
$$

\section{Experimental results}

Comparing three values obtained with the same initial height of the striker enables to evaluate the accuracy of measurement and eliminate random effects which threaten to compromise the results. The acceleration values from the accelerometer are compared in Fig. 4.

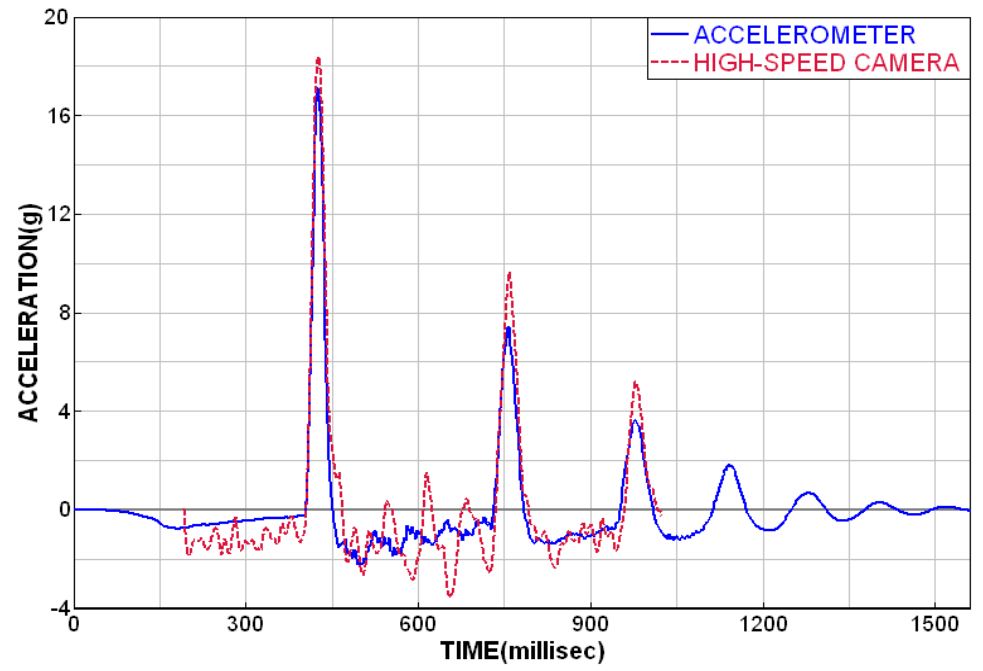

Fig. 4. Comparison of experiment results based on data from the accelerometer and high-speed camera for the initial height of $880 \mathrm{~mm}$

Initial derivative calculations were carried out using the MS Excel tool. The basis here is the definition of derivative, i.e. the most straightforward method of numerical differentiation [6] expressed by the relationship (3). The Visual Viewer tool has also been useful for data filtering and other manipulations.

$$
f^{\prime}(x)=\lim _{h \rightarrow 0} \frac{f(x+h)-f(x)}{h} \approx \frac{f(x+h)-f(x)}{h}
$$

The comparison between accelerometer readings and high-speed camera data is interesting. The simplest alternative is differentiation of the displacement from the highspeed camera data, and integration of the accelerometer readings. If displacement is considered as the result of horizontal and vertical displacement components, the speed profile calculated from the accelerometer readings should match the speed profile calculated from the displacements obtained from the high-speed camera data. In every frame, the high-speed camera detects the position with a certain accuracy which is related to the correct identification of the position of the point of interest. It introduces a small error in every frame which has no significant effect on the general trend. However, this error can be positive at one point and negative at the next one. Numerical solution of the second derivative of this function generates noise due to these small fluctuations. Hence, the displacement function appears very smooth but its second derivative is affected by 
considerable noise. Filtering of results is an effective solution here. The resulting acceleration may be affected by this filtering but both speed profiles with filtered results from the high-speed camera suggests a good correlation between both measuring methods.

\section{Discussion of results}

The material data is first validated at a higher speed. Figure 5 shows the results of the validation and calculation. Clearly, the behaviour of the non-validated material is rather close to the experimental findings. The deformation rate at the point of impact can be estimated with the aid of the initial striker velocity and the initial height of the part (4).

$$
\dot{\epsilon}(t)=\frac{d \epsilon}{d t}=\frac{d}{d t}\left(\frac{L-L_{0}}{L_{0}}\right)=\frac{v(t)}{L_{0}}
$$

Where $\epsilon$ denotes engineering strain, $\mathrm{L}_{0}$ is the initial width of the foam, $\mathrm{L}$ is the instantaneous width of the foam, and $v(t)$ is the instantaneous rate of compression of the foam at time t. Figure 5 shows results of the calculation for the initial speed of $3.9 \mathrm{~m} / \mathrm{s}$. The behaviour of the model is close to the experimental findings. By adjusting the material parameters, much better correlation can be obtained. This is clear from Fig. 5 which compares the non-validated profile and the validated profile where the latter overlaps with the experimental results at some points.

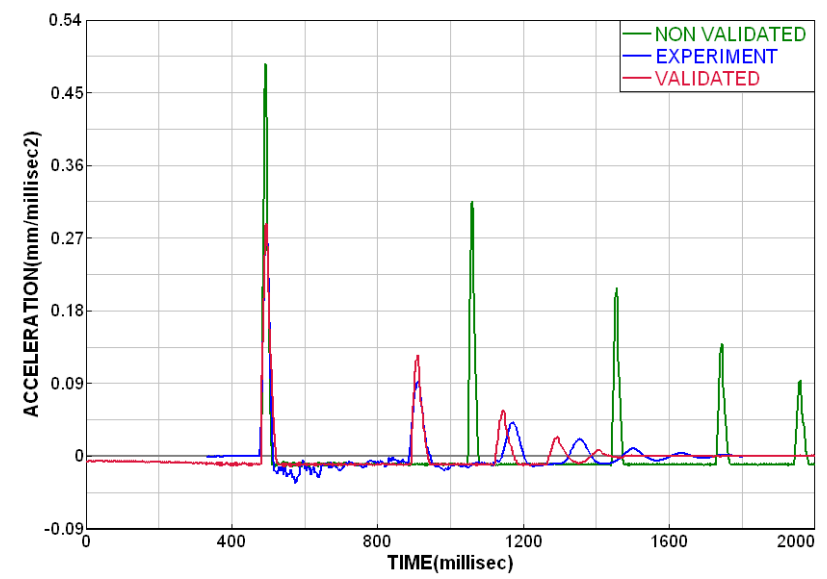

Fig. 5. Impactor acceleration during the fall from the initial height of $880 \mathrm{~mm}$

Figure 6 shows results of the calculation for the initial speed of $2.8 \mathrm{~m} / \mathrm{s}$. It is obvious that at lower speeds, the simulation results are less close to the experimental findings than at higher speeds. Nevertheless, the priority is to validate the material model for the higher speed. 


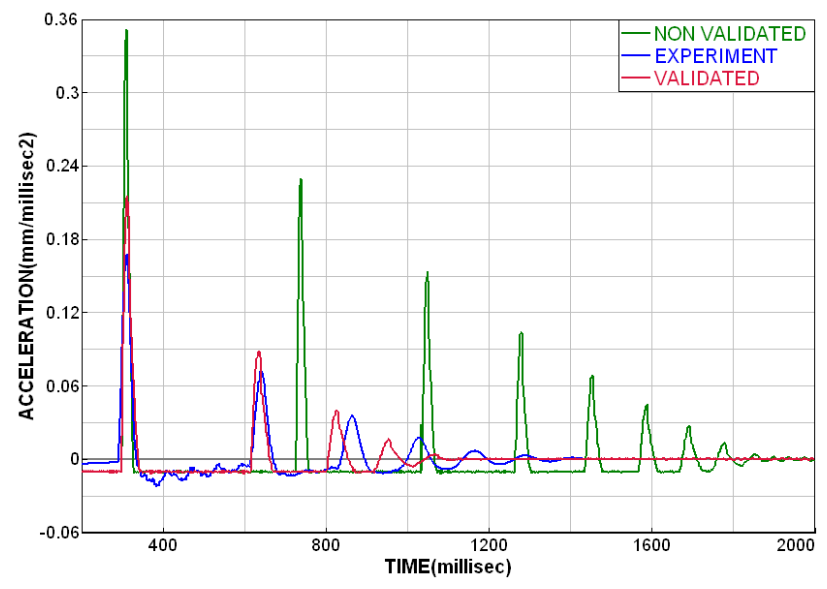

Fig. 6. Striker acceleration from the initial height of $500 \mathrm{~mm}$

The most important outputs are the parameters of foam material model for foam seat padding made from Elastoflex W 5662 covered with Holdsworth BHLH 31634. The material model validated by experiments is suitable for seat padding in explicit simulations. The used stress-strain characteristics is shown in the figure 7. Young modulus is here $\mathrm{E}=0.02 \mathrm{GPa}$, the density $\rho=4 \mathrm{e}-7 \mathrm{~kg} / \mathrm{mm} 3$ and dissipation unloading factor is UNLFAC $=0.25$. The example of material model definition with conventions of SW PamCrash is in Table 1.

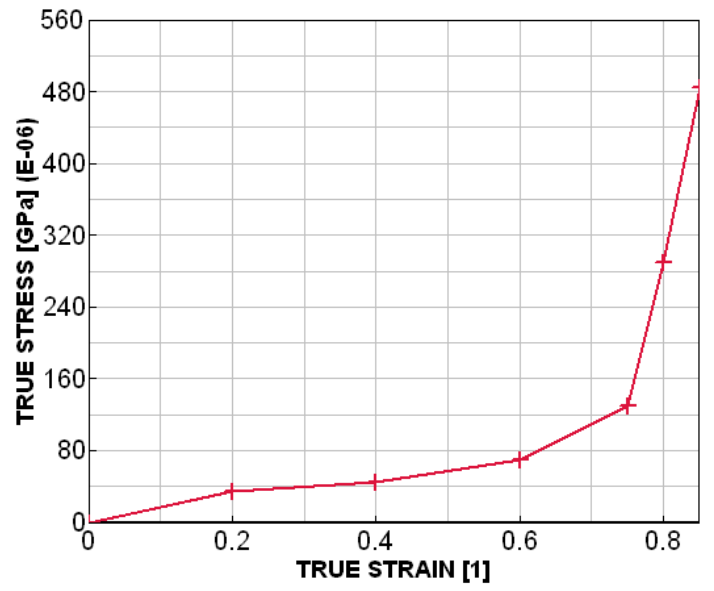

Fig. 7. The stress-strain dependence of the foam material (PUR)

Table 1. Material model parameters with conventions of SW Pam-Crash

\begin{tabular}{|c|c|c|c|l|l|}
\hline UNIT & {$[\mathbf{M M}]$} & [KG] & [MS] & [KELVIN] & \\
\hline MATYP & RHO & ISINT & ISHG & ISTRAT & \\
\hline 45 & $4 \mathrm{E}-7$ & 3 & 2 & 0 & \\
\hline QVM & IDMPD & & & & \\
\hline 1 & 0 & & & & \\
\hline E & UNLFAC & HYDRO & KSI & SLMULT & Fo \\
\hline 0.02 & 0.25 & 0. & 0.7 & 0. & 3.0 \\
\hline Q1 & Q2 & Q3 & ERATc1 & ERATc2 & ERATt1 \\
\hline 1.2 & 0.06 & 0.01 & 0 & 1 & 0 \\
\hline LCc1 & & LCc2 & & LCt1 & \\
\hline
\end{tabular}




\begin{tabular}{|c|c|c|c|l|l|}
\hline $\mathbf{X}$ & $\mathbf{Y}$ & $\mathbf{X}$ & $\mathbf{Y}$ & $\mathbf{X}$ & $\mathbf{Y}$ \\
\hline 0. & 0. & 0. & 0. & 0. & 0. \\
\hline 0.2 & $3.5 \mathrm{E}-5$ & 0.2 & $9.54 \mathrm{E}-5$ & 0.2 & $3.16 \mathrm{E}-5$ \\
\hline 0.4 & $4.5 \mathrm{E}-5$ & 0.4 & 0.000111 & 0.4 & 0.000414 \\
\hline 0.6 & $7.0 \mathrm{E}-5$ & 0.6 & 0.000159 & & \\
\hline 0.75 & 0.00013 & 0.75 & 0.000254 & & \\
\hline 0.8 & 0.00029 & 0.8 & 0.000446 & & \\
\hline 0.85 & 0.000485 & 0.85 & 0.00159 & & \\
\hline
\end{tabular}

\section{Conclusion}

The most significant result is the material model (Elastoflex W 5662 covered with Holdsworth BHLH 31634) prepared for SW Pam-Crash (see Tab 1). The material model validated by experiments is suitable for seat padding in explicit simulations. The present experiment enables us to assess whether the behaviour of a material model corresponds to the reality. In addition, the material model data can be adjusted to obtain better correlation between collision simulation and the real-world situation. To validate foamed material data, an FEM calculation was carried out, which effectively simulates the experiment. A model of a striker (a rigid body) has been created. The striker model is attached by means of a 1D element to a kinematic joint with a single degree of freedom. These bodies with weights identical to those of the real experimental parts represent the pendulum. The striker hits a cuboid created with solid eight-node elements. This cuboid represents a model of the relevant part of the seat made of a foamed material. Its height is clearly defined (by the height of the part). The horizontal size of the cuboid is increased to the point, at which further increases have no impact on the calculation. If further increases have no impact on the calculation, there is no need for simulating the entire part. The behaviour of the model is close to the experimental findings. By adjusting the material parameters, much better correlation can be obtained. This is clear from Fig. 5 which compares the non-validated profile and the validated profile where the latter overlaps with the experimental results at some points.

The present contribution was prepared under financial support of the project LO1502 'Development of the Regional Technological Institute' carried out under the auspices of the National Sustainability Programme I of the Ministry of Education of the Czech Republic aimed to support research, experimental development and innovation.

\section{References}

1. M. Avalle, G. Belingardi, R. Montanini, Characterization of polymeric structural foams under compressive impact loading by means of energy-absorption diagram. International Journal of Impact Engineering, 455-472, (2001)

2. M. Paulino, F. Teixeira-Dias, On the Use of Polyurethane Foam Paddings to Improve Passive Safety in Crashworthiness Applications. InTech, (2012)

3. Author's team of ESI Group, Virtual Performance Solution 2010. ESI Group, Paris, (2010)

4. Author's team of ESI Group, Lerning Solutions PAM-CRASH TRAINING. ESI Group, Paris, (2010)

5. Advanced Technology Group: iSPEED 2 [online]. 2013 [cit. 2013-09-6]. [WWW]: http://www.atg.cz/cz/pdf/katalogy/VT/ispeed.pdf

6. K. Kopecky: Numerical Differentiation. LECTURE NOTES FALL 2007 
7. L. Hyncik, J. Manas, J. Spicka, S. Spirk, L. Kovar, Development of 6 years old child virtual model by automatic scaling. SAE Technical Papers, SAE $10^{\text {th }}$ International Conference on Automotive Engineering 2, (2014) 